\title{
Intramural Bladder Endometriosis Secondary to Cesarean Section
}

\author{
Rathod $\mathrm{K}^{\mathbf{1}}$, Jakes $\mathrm{A}^{\mathbf{1}}$, Taylor $\mathrm{C}^{\mathbf{3}}$, Holland $\mathrm{T}^{\mathbf{2}}$ and Kunde $\mathrm{K}^{\mathbf{2}}$ \\ ${ }^{1}$ Senior Clinical Fellow, Guys and St Thomas Hospital, London, United Kingdom \\ ${ }^{2}$ Consultant Urologist, Guys and St Thomas Hospital, London, United Kingdom \\ ${ }^{3}$ Consultant Gynecologist, Guys and St Thomas Hospital, London, United Kingdom \\ *Corresponding Author: Rathod K, Senior Clinical Fellow, Guys and St Thomas \\ Hospital, London, United Kingdom.
}

DOI: 10.31080/ASWH.2020.02.0085
Received: January 14, 2020

Published: January 29, 2020

(C) All rights are reserved by Rathod K., et al.

\begin{abstract}
Endometriosis is a disease with causes still unclear, affecting approximately $15 \%$ of women of reproductive age. According to direct implantation theory, the endometrial or decidual tissues start to grow in susceptible individuals when implanted at new sites. $1-2 \%$ of women with endometriosis have involvement of the urinary tract, most frequency the bladder which is observed in up to $85 \%$ of the cases. The most effective treatment is partial cystectomy, especially via video laparoscopy. We report a case in which a patient presented with symptoms suggestive of bladder endometriosis secondary to cesarean section. It was completely excised through laparoscopy and the patient had complete resolution of symptoms and improved quality of life.
\end{abstract}

Keywords: Laparoscopy; Bladder; Urology; Endometriosis

\section{Introduction}

Endometriosis is a benign disease defined by the presence of ectopic endometrial glands and stroma, associated with pelvic pain and subfertility. The urinary bladder is involved in $70-85 \%$ of all cases of urinary tract endometriosis, followed by the ureter [1]. Deep infiltrating endometriosis by definition involves more than $5 \mathrm{~mm}$ of the peritoneum and has a prevalence of $1 \%$ in menstruating women. This condition involves the urinary bladder in 18\%$55 \%$ of cases [2] It presents with lower urinary tract symptoms such as dysuria, frequency or haematuria [3]. Inadvertent direct transplantation of endometrial tissue at the time of caesarean section or other pelvic surgery would seem the most plausible explanation for endometriosis found at this site [3].

It is seen in the context of generalized pelvic endometriosis disease or following pelvic surgical procedures such as a Caesarean section. The presence of at least one other site involved (superficial peritoneal implants, ovarian endometriomas, and extravesical deep peritoneal endometriosis) has been documented in approximately
$90 \%$ of cases with bladder endometriosis. Clinical management can be conservative, medical (e.g. hormonal therapies), or surgical. Medical treatment is invariably unsuccessful, with patients requiring surgical excision of the endometriosis nodules. We present a case of an isolated secondary intramural bladder endometriosis, which may have occurred most probably due to a previous caesarean section.

\section{Case Report}

A 46 years old woman Caucasian woman presented to the gynaecology clinic with a long-standing history of dysmenorrhea and menorrhagia. She had a normal body mass index. She also had cyclical dysuria, hematuria and symptoms of overactive bladder following her emergency caesarean section which was performed 15 years ago.

She had attended the urology clinic a few years ago with similar symptoms and was diagnosed with a bladder hamartoma on cystoscopy, which was excised. Subsequently she underwent annual outpatient flexible cystoscopies as a follow up and a nodular lesion 
was biopsied and diagnosed as endometriosis on histopathology. She had a retrograde cystogram in urology clinic, which confirmed a good bladder capacity of $500 \mathrm{ml}$. She was subsequently referred to the endometriosis clinic.

She had a 2D pelvic ultrasound scan by a gynecologist with expertise in pelvic ultrasound for endometriosis, which revealed a hypo echoic mass with filling defect measuring $32 \times 25 \times 12 \mathrm{~mm}$ affecting the entire thickness of the bladder dome. There was no vascularization on color Doppler.

After Multidisciplinary team discussion she underwent a joint procedure between the gynecologists and urologists for removal of bladder endometriosis. Cystoscopy confirmed the presence of endometriosis at the bladder dome measuring $3 \mathrm{~cm}$ in size. The urologist inserted bilateral ureteric stents before laparoscopy was performed. Laparoscopy was performed with $10 \mathrm{~mm}$ primary port at umbilicus with $0^{0}$ telescope and three $5 \mathrm{~mm}$ secondary ports. Thunder beat was used as an energy device. Retrograde filling of bladder was performed which laparoscopically confirmed the presence of a mass at the bladder dome measuring $3 \mathrm{~cm}$ in size and it also corresponded to the approximate site of caesarean section scar on the inside of the abdomen. The retropubic space of Retzius was opened first and ventral part of bladder was mobilized. A concentric incision with the thunder beat energy device was made around the endometriosis nodule and resection of the entire thickness nodule was done with clear margins. She had insertion of mirena intra-uterine system to prevent recurrence of endometriosis. The bladder was repaired in 2 layers with 2/0 V-loc barbed suture. Bladder integrity was checked with normal saline which confirmed no leaking from the suture site. The endometriosis nodule was taken out through an extended $3 \mathrm{~cm}$ incision in one of the secondary ports. A 14 Fr catheter was left in situ for 2 weeks followed by a retrograde cystogram, which did not show any leak from the suture site.

The patient was followed up in the gynecology clinic and she reported a remarkable improvement in her symptoms and quality of life. Currently, she is having six monthly follow up and will be discharge soon to the primary care.

The histology showed endometrial-type stroma and endometrial-type glands with mild hemorrhage in lamina propria with positive staining for PAX8 (endometrial glands) and D10 (endometrial stromal cells) and no evidence of malignancy.

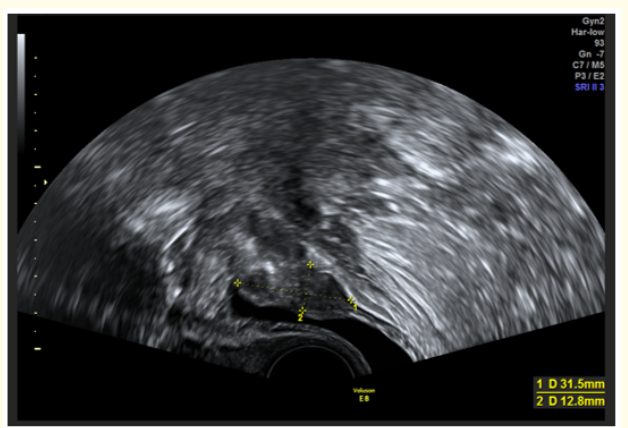

Figure 1: Endometriosis nodule in Bladder wall, sagittal view measuring $31.5 \mathrm{~mm} \times 12.8 \mathrm{~mm}$.

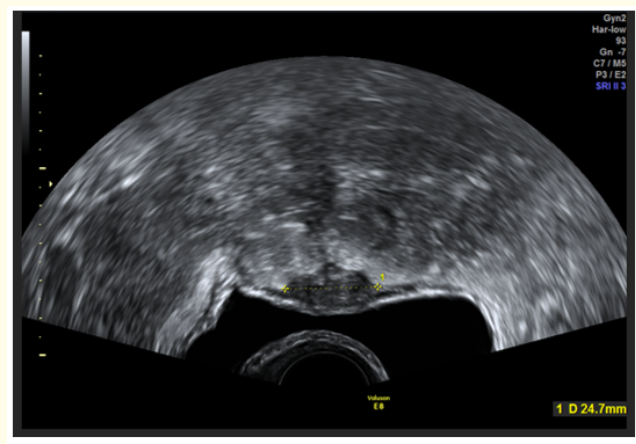

Figure 2: Endometriosis nodule in Bladder wall, coronal view measuring $24.7 \mathrm{~mm}$.

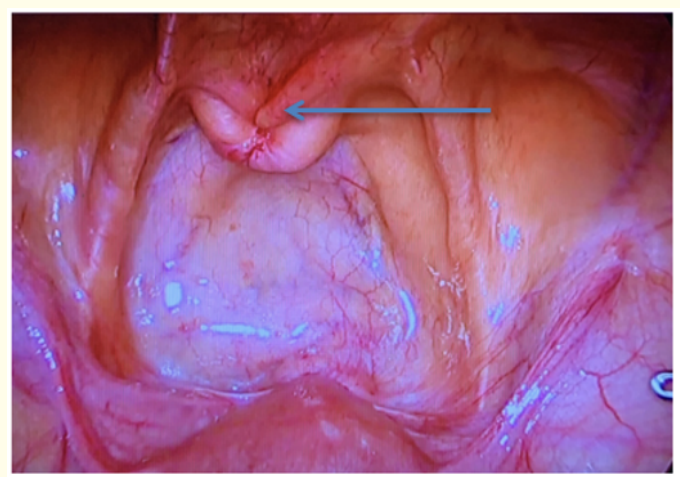

Figure 3: Laparoscopy view showing endometriosis nodule marked with an arrow. 


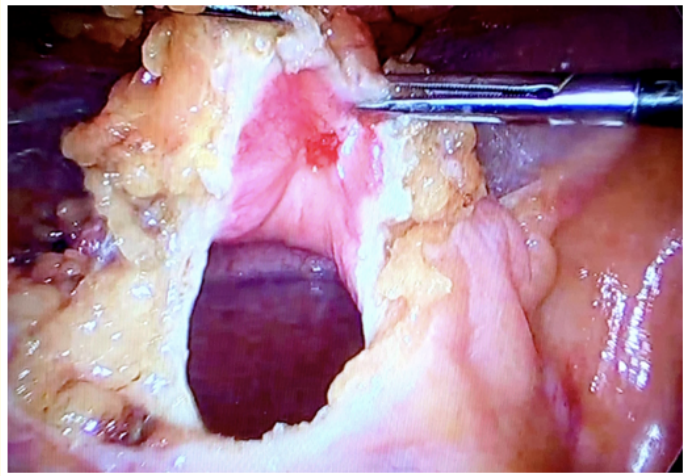

Figure 4: Laparoscopy view showing endometriosis nodule being excised.

\section{Discussion}

Our case report suggests that bladder endometriosis can be very efficiently treated with laparoscopy. Our patient underwent caesarean section 15 years ago which likely lead to the inadvertent direct transplantation of endometrial tissue. She was under the care of the urologist for more than 2 years with unexplained dysuria and hematuria with a diagnosis of a bladder hamartoma. The correct diagnosis was only achieved following a repeat cystoscopy and further biopsy of a nodular lesion.

Diagnosis of bladder endometriosis is difficult, due to vague clinical signs and symptoms, and none of them pathognomonic. The most frequent clinical signs are pelvic pain (dysmenorrhea) and infertility associated with urological symptoms (dysuria, suprapubic pain, lower tract irritation, cystitis, etc.) [4,5].

Vaginal examination did not pick up endometriosis nodule in our case. There are cases reported where examination has picked up nodule on examination. The best time to pick up endometriosis nodule on examination would be premenstrual or during menstruation [6].

Ultrasonography is the primary investigation in every case presenting with pelvic pain. It plays a vital role in diagnosing bladder endometriosis in patients presenting with cyclical dysmenorrhea, dysuria, hematuria and in the diagnosis of bladder endometriosis and in planning the most appropriate treatment [7]. Bladder endometriosis appears as a filling defect of the bladder dome with a variable protrusion into the lumen, with an iso/hypoechoic aspect without vascularization on a background of anechoic urine as seen in figure 1 and figure 2 in our case.

MRI may help guide surgical approaches, especially for deep infiltrating endometriosis and other unusual sites of presentation. Both ultrasound and MRI may suggest endometriosis, but given the significant cost differential between MRI and ultrasound, MRI is most useful for ultrasonographically-indeterminate pelvic masses [8]. There is a characteristic hyper intensity on T1 weighted images and hypointensity on T2 weighted images. We are privilege to have a gynaecologist trained in the diagnosis of deep endometriosis on ultrasound in our hospital and hence ultrasound is the primary diagnostic tool in out unit. It can vary according to different units and MRI would be superior in that case for diagnosis.

Cystoscopy with biopsy is the gold standard one-stop diagnostic test for transmural endometriosis. Endometriotic lesions progress from the serosal layer of the bladder towards the mucosa; and are seen as red or bluish lesion on cystoscopy [9].

Endometriosis is a progressive disease. It is difficult to predict in which women it will progress. Medical treatment is not effective, such as analgesia and hormones, though it can help with symptoms to buy some time before curative surgery. Definitive treatment of bladder endometriosis, which is classed as deep infiltrative disease, relies mostly on its complete excision. Proper planning is needed prior to the surgery. Medical treatments are more palliative and do not provide definitive long term care $[10,11]$.

Transurethral resection can be performed but it is associated with higher rates of recurrence and dissatisfaction [12]. Laparoscopic partial cystectomy is the gold standard as it has advantages of quicker recovery and also 95-100\% symptom remission with lower rates of relapse $[13,14]$.

\section{Conclusions}

Iatrogenic bladder endometriosis also known as secondary bladder endometriosis following a caesarean section is a rare condition. As the rate of caesarean section rises in the UK, the incidence of secondary bladder endometriosis may rise and a gynaecologist should rule out bladder endometriosis in patients with symptoms.

Cystoscopic-assisted laparoscopic surgical excision of bladder endometriosis nodules should be performed if pain persists de- 
spite medical treatment and the primary goal of the surgery should be complete removal of the bladder nodule for complete resolution of symptom. The interaction between gynecologists and urologists is relevant for the best treatment of this disease.

\section{Conflict of Interests}

The authors declare that there are no conflicts of interest regarding the publication of this paper.

\section{Bibliography}

1. Knabben L., et al. "Urinary tract endometriosis in patients with deep infiltrating endometriosis: prevalence, symptoms, management, and proposal for a new clinical classification". Fertility and Sterility 103 (2015): 147-152.

2. Koninckx PR., et al. "Deep endometriosis: definition, diagnosis, and treatment". Fertility and Sterility 98 (2012): 564-571.

3. Taff L and Jones S. "Cesarean scar endometriosis. A report of two cases". The Journal of Reproductive Medicine 47.1 (2002): 50-52.

4. Aldridge KW, Burns JR, Singh B. Vesical endometriosis: a review and 2 case reports". Journal of Urology 134.3 (1985): 539-541.

5. Shook TE and Nyberg LM. "Endometriosis of the urinary tract". Urology 31.1 (1988): 1-6.

6. Sircus SI., et al. "Bladder detrusor endometriosis mimicking interstitial cystitis". Urology 32.5 (1988): 339-342.

7. Guerriero S., et al. "Systematic approach to sonographic evaluation of the pelvis in women with suspected endometriosis, including terms, definitions and measurements: a consensus opinion from the International Deep Endometriosis Analysis (IDEA) group". Ultrasound in Obstetrics and Gynecology 48.3 (2016): 318-332.

8. Ballyguier C., et al. "Comparison of magnetic resonance imaging and transvaginal ultrasonography in diagnosing bladder endometriosis". Journal of the American Association of Gynecologic Laparoscopy 9.1 (2002): 15-23.

9. Vercellini P., et al. "Bladder detrusor endometriosis: clinical and pathogenetic implications". Journal of Urology 155.1 (1996): 84-86.

10. Koninckx PR and Martin DC. "Surgical treatment of deeply infiltrating endometriosis". In: Shaw RW (ed.) Endometriosis. Current Understanding and Management. Oxford, Blackwell Scientific (1995): 264-281.
11. Wingfield M and Healy DL. "Endometriosis: medical therapy". Baillière's Clinical Obstetrics and Gynaecology 7.4 (1993): 813838.

12. Garry R. "Endometrial ablation and resection: validation of a new surgical concept". British Journal of Obstetrics and Gynaecology 104 (1997): 1329-1331.

13. Chapron $\mathrm{C}$ and Dubuisson JB. "Laparoscopic management of bladder endometriosis". Acta Obstetricia et Gynecologica Scandinavica 78 (1999): 887-890.

14. Nezhat CH., et al. "Laparoscopic management of 15 patients with infiltrating endometriosis of the bladder and a case of primary intravesical endometrioid adenosarcoma". Fertility and Sterility 78 (2002): 872-875.

\section{Assets from publication with us}

- Prompt Acknowledgement after receiving the article

- Thorough Double blinded peer review

- Rapid Publication

- Issue of Publication Certificate

- High visibility of your Published work

Website: https://www.actascientific.com/

Submit Article: https://www.actascientific.com/submission.php Email us: editor@actascientific.com

Contact us: +919182824667 